\title{
Structure of Gibbs States of one Dimensional Coulomb Systems
}

\author{
Michael Aizenman ${ }^{1 \star}$ and Philippe A. Martin ${ }^{2}$ \\ 1 Joseph Henry Laboratories, Physics Department, Princeton University, Princeton, N.J. 08544, USA \\ 2 Laboratoire de Physique Théorique, Ecole Polytechnique Fédérale de Lausanne, \\ CH-1001 Lausanne, Switzerland
}

\begin{abstract}
We present a method of computing the Coulomb forces on particles in an infinite configuration of charges in one dimension. The resolution of the apparent nonuniqueness in this problem leads to a structural proof of the translation symmetry breaking in jellium, at all temperatures, and to a related phenomenon of phase nonuniqueness in the two component system. The appropriate generalizations of the DLR and KMS conditions for these states are discussed.
\end{abstract}

\section{Introduction}

Coulomb systems are of interest even in one dimension since:

1) Coulomb forces play such a fundamental role in Nature.

2) The systems offer tractable examples of situations in which the infinite range of the interaction poses difficulties already in the formulation of the laws of motion and of the conditions which are generally used to describe equilibrium states (KMS, DLR). The resolution of these difficulties requires the introduction of new methods which take into account the collective effect of the bulk system.

3) Some of the systems exhibit symmetry breaking and others may exist in various phases. The two component systems will be shown to admit states with a non vanishing electric field. Our method offers a unified treatment of this phenomenon and of the translation symmetry breaking in jellium, which in $[1,2]$ was proven to form a Wigner-lattice.

The one dimensional Coulomb interaction energy of particles with charges $\sigma_{i}$ located at $q_{i} \in \mathbb{R}$, is :

$$
H(q, \sigma)=-1 / 2 \sum_{i, j} \sigma_{i} \sigma_{j}\left|q_{i}-q_{j}\right|,
$$

with the corresponding electric field at $x \in \mathbb{R}$ :

$$
E(x ; q, \sigma)=\sum_{j} \sigma_{j} \operatorname{sgn}\left(x-q_{j}\right) .
$$

* Supported in part by U.S. National Science Foundation, grant No. PHY-78 25390 A01 
We shall consider the following two types of systems :

1) Two component systems, in which the charges take only the values $\sigma_{i}= \pm e$.

2) Jellium, consisting of $-e(e>0)$ charged particles immersed in a uniformly charged positive background.

Equilibrium states of finite two component systems are described by the Gibbs distribution

$$
\frac{1}{(\text { Norm. })} z^{N} e^{-\beta H(q, \sigma)} \frac{d q_{1} \ldots d q_{N}}{N !}
$$

which has to be summed over all $N$ and charge assignments of $\sigma_{i}= \pm e$.

Limits of such ensembles with the neutrality constraint were studied by Edwards and Lenard [3] and Lenard [4]. They provided a very useful form for the system's correlation functions. Additional information about the approach to the limit is contained in the work of Fröhlich and Park [5].

While the correlation functions in principle provide all the information about the local behaviour in large systems, a number of properties, and questions, are more naturally described in the phase space formalism [6]. In it, the same state is represented by a probability measure on the phase space of infinite configurations of charged particles on a line. We denote by $\Omega$ this space whose points, $\omega$, may be regarded as equivalence classes, with respect to relabeling, of sequences $\left\{\left(q_{i}, \sigma_{i}\right)\right\}_{i \in \mathbb{Z}}$. States of the system can now be described by probability measures on $\Omega$ which we denote by $\mu$. We should also use $\mu(f)$, or simply $\langle f\rangle$, expectation value of a function $f(\omega)$ on $\Omega$.

By the finiteness of the density of particles in the system [3] we know that typical configurations $\omega \in \Omega$ have only finite occupation in any bounded interval. In this setup, we now pose the first problem which bears on the physics of the system.

\section{Question 1}

What are the forces on the particles in a completely specified configuration $\omega=\left\{\left(q_{i}, \sigma_{i}\right)\right\}$ ?

In contrast with forces due to short range interactions, the one dimensional Coulomb force does not decrease with the separation, which renders the usual summation meaningless. In fact, the problem may seem to be deeper than that of a good summation procedure. The infinite system formalism has been devised for the study of local behavior as an approximation to a finite system which, on the scale of interparticle distance, is extremely large. However, since all the charges do contribute directly to the local field, it is not a-priori clear whether in this formalism there is no loss of important information about charges whose distance is of the order of the system's length. Nevertheless, we shall see that the existent effect of the bulk system can be computed quasilocally from the positions of the charges.

The above problem is related to another question which bears on the structure of the general formalism. 


\section{Question 2}

What local condition of the states reflects their equilibrium character?

The standard Dobrushin-Lanford-Ruelle condition $[7,8]$ is that the density in any region $\Lambda$, conditioned on the configuration outside of that set, is given by a corresponding grand-canonical ensemble, with the energy function which takes into account also the interaction with the outer particles. To state this condition one has to make sense of the electric potential throughout the system, which leads to even worse summation problems than those with the electric field.

Our method would yield an answer to this question, revealing an interesting feature. Namely, for typical configurations, once the particles outside a region $\Lambda$ are specified the total charge in $\Lambda$ is sharply defined. This is related to the surpression of charge fluctuations discussed in [9]. Non-normal fluctuations have also been found by Dyson and Mehta [10] in the distribution of eigenvalues of random matrices, which is given by a Gibbs state with the logarithmic potential. They suggested an application of the understanding of such an effect to the study of nuclear interactions.

\section{Electric Field Ensembles for two Component Systems}

The first of the two problems mentioned above can be restated as the question of a quasi-local inversion of the correspondence

$$
\text { electric-field } \rightarrow \text { charge configuration . }
$$

For finite systems, where it certainly is well defined, the electric field is piecewise constant and has jump discontinuities, whose size is twice the charge, at the particles locations (see Fig. 1). Thus the charge configuration is a local function of the electric field. In the other direction, however, the correspondence is not local. A uniform shift of $E(x)$ by a constant inside a large interval corresponds to a change in only the boundary charge. In the thermodynamic limit one may expect that two electric field configurations which differ only by a constant would correspond to the same charge configuration. A key observation in the following method is that in this limit the relation (2.1) is, nevertheless, invertible when restricted to typical configurations, i.e. after excluding a set whose measure is zero.

In view of the local and unique correspondence (2.1), and regardless of its inversion, the statistical distribution of the electric field completely determines the state of the system of charges. This observation was used by Lenard [4], who thus

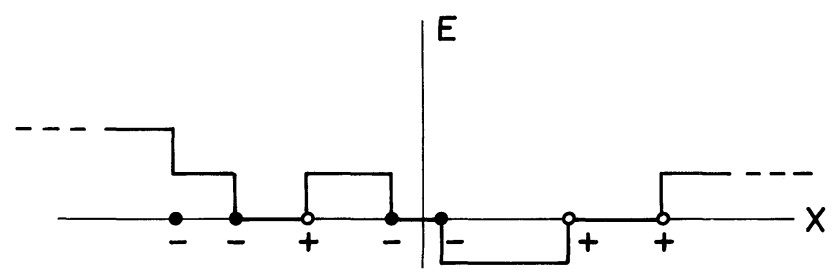

Fig. 1. The electric field in the relation to a charge configuration 
studied the limiting state of finite systems which were constrained to be neutral. He found the following result:

Proposition 1. For any $\beta, z>0$ the distribution of the electric field in the ensemble (1.3) with the constraint $\sum_{i} \sigma_{i}=0$, locally converges to a limit. In this limit:

1) $E(x)$ takes values in $2 e \mathbb{Z}$, and typical configurations of $E(\cdot)$ are piecewise constant with discrete jumps of $\pm 2 e$.

2) The probability distribution of $E(\cdot)$ has the Markov property and is translation invariant, and mixing with respect to translations.

More specifically, if the physical position, $x$, is viewed as a "time" variable, $E(x)$ has the distribution of the path of a Markov random process. The corresponding semigroup, on $\ell^{2}(2 e \mathbb{Z})$, is generated by

$$
\mathscr{L}=-z \Delta+\frac{\beta}{4} \breve{E}^{2}
$$

with the discrete Laplacian, $\Delta$, defined by:

$$
(\Delta \phi)(u)=\phi(u+2 e)+\phi(u-2 e)-2 \phi(u)
$$

and the multiplication operator:

$$
(\check{E} \phi)(u)=u \phi(u) \text {. }
$$

The invariant distribution of the electric field at a site $x$ is

$$
P(E(x)=u)=\psi(u)^{2},
$$

where $\psi$ is the eigenvector of $\mathscr{L}$ in $\ell^{2}(2 e \mathbb{Z})$ corresponding to its minimal eigenvalue there, $\lambda$. The probability kernel of the process is

$$
P(E(x)=u \mid E(0)=v)=\psi(u) e^{-x(\mathscr{L}-\lambda)}(u, v) \psi(v)^{-1}
$$

for any $x \geqq 0$.

A major reason why the electric field, $E$, is a convenient variable for the system is that its fluctuations are controlled by the Gibbs factor. This is due to the well known electrostatic identity, which relates the energy of a system of charges $\sigma_{i}$ located at $q_{i} \in I_{L}=[-L / 2, L / 2]$ to a positive quadratic form in $E$ :

$$
H(q, \sigma)=1 / 4 \int_{I_{L}} d x|E(x)|^{2}-1 / 4\left(\sum_{i} \sigma_{i}\right)^{2} L
$$

Another convenient feature is the simplicity of the measure on the electric field ensembles which corresponds to the distribution of non-interacting particles. Namely, it is the shift invariant measure $v(d E)$ on the space of paths of a random jump process on $2 e \mathbb{Z}$, which is generated by $-z \Delta$, for which $v(\{E(0)=2 e k\})=1$, $\forall k \in \mathbb{Z}$. With respect to $v$, the probability for $E(\cdot)$ to have a jump discountinuity of $2 e$ or of $-2 e$ in an interval $d x$, are each of the order $z d x$, independently of the jumps made outside of $d x$. The probability to have no jump in an interval of length $x$ is thus $e^{-2 z x}$. Therefore, the $v$-measure of paths whose discountinuities in $I_{L}$ 
occur at $d q_{i}$ and are of the sizes $2 \sigma_{i}(= \pm 2 e)$ is, (ignoring infinitesimals in the exponents

assuming $q_{1} \leqq q_{2} \leqq q_{3} \ldots$

$$
e^{-2 z q_{2}} z d q_{1} e^{-2 z\left(q_{2}-q_{1}\right)} z d q_{2} \ldots=e^{-2 z L} \prod_{i}\left(z d q_{i}\right)
$$

The measure $v(d E)$ reproduces therefore the (Liouville-Lebesgue) a-priori distribution of the charges. The neutrality constraint corresponds to conditioning on $E\left(\frac{L}{2}\right)=E\left(-\frac{L}{2}\right)=0$.

Placing these elements together, we see that in the above finite volume neutral ensembles the expectations of local functions of $E$ can also be written as:

$$
\mu_{0}^{L}(f)=\frac{1}{(\text { Norm. })} \underset{E\left(\frac{L}{2}\right)=E\left(-\frac{L}{2}\right)=0}{ } v(d E) \exp \left(-\frac{\beta}{4} \int_{I_{L}} d x(E(x))^{2}\right) f(E) .
$$

Proposition 1 follows from this formula using the Feynman-Kac prescription [11] and some properties of $\mathscr{L},[4,12]$. We provide the complete details for the last step in this argument in the proof of Proposition 2.

The existence of a well defined limit for the states of $E(\cdot)$ reduces Question 1 to the problem of reconstruction of $E(\cdot)$ from the position of its discontinuities which are the locations of the charges. Technically the question makes no a-priori sense in the particle phase-space formalism, where $E(\cdot)$ has no clear definition. As we shall now see, the reconstruction can be done, despite the apparent nonuniqueness, by a method which gives the right result for typical configurations. We shall state the result in the particle phase $\Omega$, with the understanding that we really deal with a problem on the space of configurations of $E(\cdot)$.

Let $\mu_{0}$ be the limiting state of neutral ensembles described in Proposition 1 . We say that a property holds almost surely, or for almost every (a.e.) $\omega \in \Omega$, if the set on which it fails has zero measure.

Lemma 1. Let $x \in \mathbb{R}$. For $\mu_{0}$-a.e. configuration $\omega \in \Omega$ the following limit exists and gives the value of $E(x)$ :

$$
E(x)=-\lim _{K \rightarrow \infty} 2 \sum_{q_{i} \in[x, x+K]} \sigma_{i}\left(1-\frac{q_{i}-x}{K}\right) .
$$

Proof. Let $y>x$. Assuming there are no particles at $x$ or $y$ :

$$
E(y)=E(x)+2 \sum_{q_{i} \in[x, y]} \sigma_{i} .
$$

By the ergodic theorem, which is applicable by Proposition 1 (2),

$$
\lim _{K \rightarrow \infty} \frac{1}{K} \int_{x}^{x+K} d y E(y)=\mu_{0}(E(0))
$$

$\mu_{0}$-almost surely.

Substituting (2.11) in (2.12) we obtain, for $\mu_{0}$-a.e. $\omega$,

$$
E(x)=-\lim _{K \rightarrow \infty} 2 \sum_{q_{i} \in[x, x+K]} \sigma_{i}\left(1-\frac{q_{i}-x}{K}\right)+\mu_{0}(E(0)) .
$$


By the charge conjugation $(\sigma \rightarrow-\sigma)$ symmetry of $\mu_{0}$ :

$$
\mu_{0}(E(0))=0
$$

which leads to (2.10).

Remark 1. A resolution of the apparent conflict between (2.10) and the nonuniqueness which we discussed above is that in each family of electric field configurations which differ only by constants, all but at most one would not occur in the equilibrium state. In terms of finite ensembles, this is related to the fact that the change in the energy produced by a shift of the electric field by a constant is of the order of the volume. The associated entropy is only of the order of the size of the boundary, since that is where the charges which produce the shift are located.

Lemma 1 answers Question 1 but also leads to the following surprising observation.

Corollary 1. For $\mu_{0}$-a.e. $\omega \in \Omega$

$$
\lim _{K \rightarrow \infty} \exp \left[2 i \pi \sum_{q_{\imath} \in[0, K]}\left(\frac{q_{i}}{K}\right)\left(\frac{\sigma_{i}}{e}\right)\right]=1 .
$$

Proof. Exponentiating (2.13) we get, after dropping trivial terms

$$
\lim _{K \rightarrow \infty} \exp \left[2 i \pi \sum_{q_{t} \in[0, K]}\left(\frac{q_{i}}{K}\right)\left(\frac{\sigma_{i}}{e}\right)\right]=\exp \left[2 i \pi\left(\frac{E(0)-\mu_{0}(E(0))}{2 e}\right)\right]
$$

for $\mu_{0}$-a.e. $\omega \in \Omega$. However, $E(0)$ takes only values in $2 e \mathbb{Z}$. This, together with (2.14), implies (2.15).

(2.15) is surprising since for each $K<\infty$ the left hand side varies continuously over the full unit circle when the charges are shifted. This suggests the following question.

\section{Question 3}

Does the two component system have other "equilibrium states" for which the left hand side of (2.15) takes, almost surely, other unitary values?

In the next section we shall discuss such states which also correspond to situations of physical interest.

\section{The $\theta$-States}

In the previous section we have discussed limits of neutral systems. A more general class of ensembles, which will also be needed to describe the local structure of the above states, is obtained by considering other values for the total charge and adding external charges as boundary conditions. It is easy to see that such ensembles in an interval $[a, b]$ are completely characterized by the values of the electric field at the boundary $E_{\ell}=E(a), E_{\imath}=E(b)$.

For specified $\left(E_{\ell}, E_{\imath}\right)$ the total charge in the interval $[a, b]$ (with $\left.a<b\right)$ is constrained by:

$$
\sum_{q_{i} \in[a, b]} \sigma_{i}=\frac{E_{\imath}-E_{\ell}}{2}=Q\left(E_{\ell}, E_{\imath}\right) .
$$


The energy of a charge configuration in $[a, b]$, with the above constraint on $E$ can be calculated from (2.7), using (2.11). A simple way of representing the answer is by adding to the system's Hamiltonian (1.1) the effect of two boundary charges: $\frac{C}{2}$ at $a$ and $-\frac{C}{2}$ at $b$, with

$$
C=\frac{E_{\ell}+E_{\imath}}{2} .
$$

We shall refer to the distribution (1.3) in $[a, b]$ with the above described Hamiltonian and the charge constraint (3.1) as the $\left(E_{\ell}, E_{\imath}\right)$-equilibrium ensemble in $[a, b]$ (omitting $\beta$ and $z$ in this terminology; all statements in the following will hold for every fixed $\beta, z>0$ ).

The main result in this section, which would also provide an answer to Question 3, is :

Proposition 2. Let $\beta, z>0$. For any given $\left(E_{\ell}, E_{\imath}\right)$ with $Q\left(E_{\ell}, E_{\imath}\right) \in e \mathbb{Z}$ (a consistency condition) the $\left(E_{\ell}, E_{\imath}\right)$-equilibrium ensembles in $\left[-\frac{L}{2}, \frac{L}{2}\right]$ converge locally, as $L \rightarrow \infty$, to a state $\mu_{\theta}, \theta \in[0,2 e]$, which depends only on $\theta=E_{\ell} / \bmod 2 e$. Further, any two states $\mu_{\theta_{1}}, \mu_{\theta_{2}}$ with $\theta_{1}, \theta_{2} \in[0,2 e), \theta_{1} \neq \theta_{2}$, are mutually singular.

Remarks. Two interesting situations to which this result refers are:

1) Ensembles with a fixed total charge, free of other boundary conditions, $\left(E_{\ell}, E_{z}\right)=(-Q, Q)$. The limiting state is different in the case the charge is odd, in units of $e$, from that of even charge, which leads to the same limit as the neutral ensemble.

2) Neutral ensembles in the presence of constant external field $D, E_{\ell},=E_{\imath}=D$. In this case the limiting state is a nondegenerate periodic function of $D$.

We shall prove Proposition 2 at the end of this section using the electric field ensembles. These are constructed similarly to the neutral ensemble discussed in Lemma 1.

Lemma 2. Let $\beta, z>0$, and let $\left(E_{\ell}, E_{\imath}\right)$ be given with $Q\left(E_{\ell}, E_{\imath}\right) \in e \mathbb{Z}$. As $L \rightarrow \infty$, the distribution of the electric field in the $\left(E_{\ell}, E_{\imath}\right)$-equilibrium ensembles in $\left[-\frac{L}{2}, \frac{L}{2}\right]$ locally converges to a limit. The limiting distribution depends only on $\theta=E_{\ell} / \bmod 2 e$ $=E_{2} / \bmod 2 e$ and has the properties listed in Proposition 1, except that the range of $E(x)$ is $2 e \mathbb{Z}+\theta$ and the relevant domain for the semigroup (and its generator) is $\ell^{2}(2 e \mathbb{Z}+\theta)$.

Proof. The range of $E(x)$ in the finite volume ensembles is clearly $2 e \mathbb{Z}+\theta$. An a-priori measure which satisfies this constraint is :

$$
v_{\theta}(d E)=v(d(E-\theta)),
$$

obtained by shifting $v$.

Repeating the argument which led to (2.9), one finds that in the $\left(E_{\ell}, E_{\imath}\right)$ equilibrium ensembles in $I_{L}$, the expectation of a local function of $E$ is:

$$
\langle f\rangle_{\left(E_{\ell}, E_{\ell}\right)}^{L}=\frac{1}{\operatorname{Norm} .} \int_{\substack{E(-L / 2)=E_{\ell} \\ E(L / 2)=E_{\ell}}} v_{\theta}(d E) \exp \left(-\frac{\beta}{4} \int_{I_{L}} d y|E(y)|^{2}\right) f(E) .
$$


Let $\left\{A_{x}\right\}_{x \geqq 0}$ be the semigroup of (Hermitian) operators on $\ell^{2}(2 e \mathbb{Z}+\theta)$ with the kernels :

$$
A_{x}(u, v)=\int_{\substack{E(0)=u \\ E(x)=v}} v_{\theta}(d E) \exp \left(-\frac{\beta}{4} \int_{0}^{x} d y|E(y)|^{2}\right) .
$$

I.e., by the Feynman-Kac formula [12], $A_{x}=\exp \left(-x \mathscr{L}_{\theta}\right)$, where $\mathscr{L}_{\theta}$ is the operator which formally looks as $\mathscr{L},(2.3)$, but acts in $\ell^{2}(2 e \mathbb{Z}+\theta)$. We shall also denote by $\chi_{u} \in \ell^{2}(2 e \mathbb{Z}+\theta)$ the indicator function:

$$
\chi_{u}(v)=\delta_{u, v} .
$$

For $f(E)=\prod_{i=1}^{N} g_{i}\left(E\left(x_{i}\right)\right)$, with some $-L / 2<x_{1}<x_{2} \ldots<x_{N}<L / 2$, we may rewrite (3.4) as :

$$
\langle f\rangle_{\left(E_{\ell}, E_{\eta}\right)}^{L}=\frac{\left(\chi_{E_{\ell}}, A_{x_{1}+L / 2} g_{1}(\check{E}) A_{x_{2}-x_{1}} g_{2}(\check{E}) \ldots g_{N}(\check{E}) A_{L / 2-x_{N}} \chi_{E_{z}}\right)}{\left(\chi_{E_{\ell}}, A_{L} \chi_{E_{2}}\right)} .
$$

While one could now deduce the lemma using the spectral decomposition and properties of $\mathscr{L}_{\theta}$, we shall prove it without using the self-adjointness. As a consequence, the following argument will also be applicable in the analysis of the jellium. With this purpose in mind, let us recall the following generalization of the Peron-Fröbenius theorem.

Theorem (Krein and Rutman [13]). Let $A$ be an operator on $\ell^{2}$, with the kernel $A(m, n)$, such that

1) $A$ is compact.

2) $A(m, n) \geqq 0 \forall m, n$.

3) For any pair $m, n$ there exists $N(m, n)$ such that for all $k>N(m, n)$

$$
\left(A^{k}\right)(m, n)>0 \text {. }
$$

Then:

1) $A$ has a real and nondegenerate eigenvalue, $\lambda(A)$, which is larger (with a positive gap) than the modulus of any other point in the spectrum.

2) $\lambda(A)=\lambda\left(A^{*}\right)$ and the corresponding eigenvectors $\psi, \psi^{\prime}$ of $A$ and $A^{*}$ are strictly positive.

3)

$$
e^{-\lambda k} A^{k} \underset{k \rightarrow \infty}{\longrightarrow} \frac{\left|\psi^{\prime}\right\rangle\langle\psi|}{\left\langle\psi \mid \psi^{\prime}\right\rangle}
$$

(in Dirac notation), uniform convergence of operators.

Assumptions 2) and 3) are clearly satisfied by any $A_{x}$ with $x>0$. We shall now prove that so is 1$)$. By (3.5)

$$
\begin{aligned}
A_{x}(u, v)= & \int_{\substack{E(0)=u \\
E(x)=v \\
2 e \# \cong w / 2}} v_{\theta}(d E) \exp \left(-\frac{\beta}{4} \int_{0}^{x} d y|E(y)|^{2}\right) \\
& +\int_{\substack{E(0)=u \\
E(x)=v \\
2 e \#>w / 2}} v_{\theta}(d E) \exp \left(-\frac{\beta}{4} \int_{0}^{x} d y|E(y)|^{2}\right),
\end{aligned}
$$


where \# denotes the number of jump discontinuities $E$ has in $[0, x]$, and $w=\max (|u|,|v|)$. Using an energy bound for the first term and an entropy bound for the second, we have

$$
\begin{aligned}
A_{x}(u, v) \leqq & \exp \left\{-\frac{\beta}{4} x\left(\frac{w}{2}\right)^{2}\right\} \\
& +\sum_{\ell>w /(4 e)} \frac{(2 z x)^{\ell}}{\ell !} e^{-2 z x} \\
\leqq & \exp \left\{-2^{-4} \beta x w^{2}\right\}+(2 z x)^{[w /(4 e)]} /[w /(4 e)] !
\end{aligned}
$$

Therefore

$$
\operatorname{tr} A_{x}^{*} A_{x}=\sum_{u, v \in 2 e \mathbb{Z}+\theta}\left|A_{x}(u, v)\right|^{2}<\infty,
$$

i.e. $A$ is in the Hilbert-Schmidt class, and hence compact.

Since $\left\{A_{x}\right\}$ is a strongly continuous semigroup (its continuity can be easily seen by an entropy estimate as the one used above), the eigenvector $\psi$ of $A_{x}$, discussed in the Krein-Rutman theorem, is an eigenvector of the generator and is independent of $x$. Similarly, (3.9) holds with $A_{x}$ substituted for $A^{k}$ and $x \rightarrow \infty$ as a continuous variable. Substituting it in (3.7) we obtain a proof of the lemma.

We note, in particular, that the limiting distribution of $E(0)$ is given by

$$
\langle\{E(0)=u\}\rangle_{\theta}=\psi_{\theta}(u) \psi_{\theta}^{\prime}(u)=\psi_{\theta}(u)^{2},
$$

where $\psi_{\theta}(u)$ is the ground state of $\mathscr{L}_{\theta}$ (self-adjoint).

We shall refer to the states on $\Omega$ which correspond to the above distributions of $E$ as the $\theta$-states, and denote them by $\mu_{\theta}$ (consistently with the previously defined symbol $\mu_{0}$ ).

The extension of Lemma 1 to these states is, in the sense explained in Sect. 2:

Proposition 3. Let $x \in \mathbb{R}, \theta \in[0,2 e)$. For $\mu_{\theta}$-a.e. configuration $\omega \in \Omega$ the following limits exist and each gives the value of $E(x)$

$$
\begin{gathered}
E(x)=-\lim _{K \rightarrow \infty} 2 \sum_{q_{i} \in[x, x+K]} \sigma_{i}\left(1-\frac{q_{i}-x}{K}\right)+\langle E(0)\rangle_{\theta}, \\
E(x)=\lim _{K \rightarrow \infty} 2 \sum_{q_{i} \in[x-K, x]} \sigma_{i}\left(1+\frac{q_{i}-x}{K}\right)+\langle E(0)\rangle_{\theta} .
\end{gathered}
$$

Proof. The derivation of (3.11) is identical to that of (2.11) except that (2.14) no longer holds. (3.12) is proven similarly.

Adapting the arguments which led to Corollary 1, we also have:

Corollary 2. Let $\theta \in[0,2 e)$, then for $\mu_{\theta}$-a.e. $\omega$ :

$$
\lim _{K \rightarrow \infty} \exp \left[2 i \pi \sum_{q_{i} \in[0, K]}\left(\frac{q_{i}}{K}\right)\left(\frac{\sigma_{i}}{e}\right)\right]=\exp \left[2 i \pi\left(\frac{\theta-\mu_{\theta}(E(0))}{2 e}\right)\right] .
$$

In order to see that in fact the $\theta$-states have the property suggested in Question 3, we need: 
Lemma 3. The function $g(\theta)=\theta-\langle E(0)\rangle_{\theta}$ defines a monotone bijection $g:[0,2 e) \rightarrow[0,2 e)$.

Proof. For any $\theta \in[0,2 e), \mathscr{L}_{\theta}$ is unitarily equivalent to the operator

$$
\hat{\mathscr{L}}_{\theta}=-z \Delta+\frac{\beta}{2}(\check{E}+\theta)^{2}
$$

on $\ell^{2}(2 e \mathbb{Z})$. The ground state of $\hat{\mathscr{L}}_{\theta}$ is given by:

$$
\hat{\psi}_{\theta}(u)=\psi_{\theta}(u+\theta) \text {. }
$$

Since $\hat{\mathscr{L}}_{\theta}-\frac{\beta}{2} \theta^{2}$ is affine in $\theta$, its lowest eigenvalue is a concave function of $\theta$. In fact it is strictly concave, since otherwise it would follow that for two distinct values of $\theta$ the operators $\hat{\mathscr{L}}_{\theta}$ have the same ground state, a possibility which can easily be contradicted. Further, for each $\alpha \in[0,2 e)$, the graph of $\left(\hat{\psi}_{\alpha}\left(\hat{\mathscr{L}}_{\theta}-\frac{\beta}{2} \theta^{2}\right) \hat{\psi}_{\alpha}\right)$ (viewed as a function of $\theta$ ) is a tangent, at $\theta=\alpha$, to the graph of inf $\operatorname{spec}\left(\hat{\mathscr{L}}_{\theta}-\frac{\beta}{2} \theta^{2}\right)$. It is a well known consequence of the strict convexity that the slope of such tangents increases in $\alpha$. By this argument (or, alternatively, using the Feynman-Heillman formula) the following function of $\alpha$ is monotone decreasing:

$$
\begin{aligned}
\left\langle\hat{\psi}_{\alpha}, \frac{d}{d \theta}\left(\hat{\mathscr{L}}_{\theta}-\frac{\beta}{2} \theta^{2}\right) \hat{\psi}_{\alpha}\right\rangle & =\left\langle\hat{\psi}_{\alpha}, \beta \breve{E} \hat{\psi}_{\alpha}\right\rangle=\beta \sum_{u \in \mathbb{Z} e} u \hat{\psi}_{\alpha}(u)^{2} \\
& =\beta\left[\sum_{u \in \mathbb{Z} e}(u+\alpha) \psi_{\alpha}(u+\alpha)^{2}-\theta\right]=-\beta g(\alpha) .
\end{aligned}
$$

Thus $g(\theta)$ is strictly increasing in $\theta$ on $[0,2 e)$. Further, the boundary values are 0 and $2 e$, since $\langle E(0)\rangle_{0}=\langle E(0)\rangle_{2 e}=0$.

Proof of Proposition 2. The existence of the limiting states and their sole dependence on $\theta$ follows, as discussed above, from Lemma 2. Their mutual singularity follows from Corollary 2 and Lemma 3, which prove that each of the $\theta$-states is supported on a set of configurations which has zero measure with respect to the other states.

Remarks. 1) The periodicity in $\theta$ of $\langle\ldots\rangle_{\theta}$ is due to screening, which in one dimension is not complete. When a system is placed in an external electric field (e.g. one produced by boundary charges), the integral part of the field, in the units of $e$, gets screened by the transfer of some of the system's charges. The total effect depends only on the unscreened fractional part of the applied field $-\theta$. This argument does not yet prove that the various $\theta$-states have different distributions of charge. That is a consequence of the reversibility of the correspondence (2.1).

2) The charge densities in the $\theta$-states are always zero. This can be shown as follows. Let $\varrho_{\theta}$ be the charge density in $\langle\ldots\rangle_{\theta}$.

Then for any $L>0$ :

$$
\varrho_{\theta}=\frac{1}{L}\left\langle\sum_{q_{i} \in[0, L]} \sigma_{i}\right\rangle_{\theta}=\frac{1}{2 L}\langle(E(L))-E(0)\rangle_{\theta}=0
$$


by the translation invariance and integrability of the distribution of $E$. However, the average electric field in a $\theta$-state is not zero, except for $\theta=0, e$. This follows from the fact that $\lambda_{\theta}$ is strictly decreasing (resp. increasing) in $(0, e)$ [resp. $\left.(e, 2 e)\right]$, [12].

3) There is another fruitful approach to the study of Coulomb systems, based on the "Sine-Gordon transform". It turns out, [14], that in this formalism the $\theta$-states offer simple analogs of " $\theta$-vacua", and related phenomena, which are studied in the context of gauge fields in higher dimensions.

\section{Jellium}

In this section we shall consider the jellium, which is a one component system of negatively charged particles, with charges $-e$, distributed over a uniformly charged background with the charge density $\varrho>0$. Among the system's interesting features is the translation symmetry breaking, which for the classical jellium was proven to occur at all temperatures $[1,2]$, and which (at low temperatures) corresponds to the formation of "Wigner-lattice".

The Hamiltonian for such a finite system in $[-L / 2, L / 2]$, with $N$ negative charges located at $\left\{q_{i}\right\}$, is

$$
H(q)=-\frac{e^{2}}{2} \sum_{i, j}^{N}\left|q_{i}-q_{j}\right|+e \varrho \sum_{i}^{N} q_{i}^{2}+c(N, L) .
$$

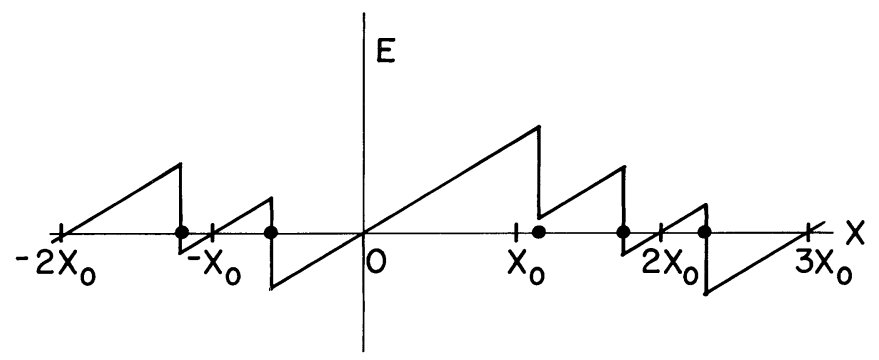

Fig. 2. The electric field in jellium

We shall consider neutral ensembles, i.e. constrained by:

$$
N=\varrho L / e .
$$

Because of the translation symmetry breaking it is now important to vary $L$ over only such values that the total positive charge in $[0, L / 2]$ is an integer in the units of $e$, i.e.

$$
L / 2 \in x_{0} \mathbb{Z} \quad \text { with } \quad x_{0}=e / \varrho .
$$

Our main result about the jellium is :

Proposition 4. For any $\beta>0$, the finite volume ensembles with the Hamiltonian (4.1) and the constraints (4.2) and (4.3) converge, as $L \rightarrow \infty$, to a limiting state which is:

1) Non invariant under continuous translations, although it is periodic with period $x_{0}=e / \varrho$ and ergodic with respect to shifts by $x_{0}$. 
Further :

2) In the limiting state for almost any configuration, $q=\left\{q_{i}\right\}$ :

$$
\lim _{y \rightarrow \infty} e^{i \pi y / x_{0}} \prod_{q_{1} \in[x, x+y]} e^{i 2 \pi\left[q_{i}-x\right) / y}=e^{i 2 \pi x / x_{0}}
$$

[(4.4) being a very explicit manifestation of the non-invariance].

In order to prove this proposition we shall follow the method explained in the previous sections. Namely, first study the electric field ensemble and then prove that the relation between $E(\cdot)$ and the charge configuration, $\left\{q_{i}\right\}$, is invertible. From this point of view the non-invariance is an obvious consequence of the following structural fact. In the neutral ensemble $E( \pm L / 2)=0$ and for any $x \leqq y$ in $[-L / 2, L / 2]$

$$
E(y)=E(x)+2 \varrho(y-x)-2 e \sum_{q_{\imath} \in[x, y]} 1,
$$

assuming there are no charges at $\{x, y\}$. Therefore the range of values of $E$ at a site $x$ is constrained by

$$
E(x) \in 2 e \mathbb{Z}+2 e x / x_{0} .
$$

This periodic constraint implies that the distribution of $E(\cdot)$ cannot be invariant under continuous translations. It should be noted that (4.6) does not yet prove that the distribution of particles is non-invariant. That would follow once we know that $E(\cdot)$ can be reconstructed from $\left\{q_{i}\right\}$.

Lemma 4. The distribution of the electric fields in the ensembles mentioned in Proposition 1 locally converges to a limit. The limiting distribution has the following properties.

1) It is periodic, with period $x_{0}$, and ergodic with respect to shifts by $x_{0}$.

2) It is Markovian.

3) $E(\cdot)$ satisfies the constraints (4.6), and almost surely has only isolated jump discontinuities of size $-2 e$.

4) With probability one, $E(\cdot)$ may be recovered from the location of its discontinuities, $\left\{q_{i}\right\}$, by:

$$
E(x)=\lim _{K \rightarrow \infty} 2 e \sum_{q_{i} \in[x, x+K]}\left(1-\frac{q_{i}-x}{K}\right)-e K / x_{0}
$$

and

$$
E(x)=\lim _{K \rightarrow \infty}-2 e \sum_{q_{i} \in[x-K, x]}\left(1+\frac{q_{i}-x}{K}\right)+e K / x_{0} .
$$

Proof. Let $v_{J}(d E)$ be the distribution on "paths" $E(x)$ which satisfy (4.6), having jump discontinuities, by $-2 e$, independently distributed with density $\varrho / e=1 / x_{0}$. Most of the discussion of Proposition 1 and the proof of Lemma 2 apply to the jellium, with $v_{J}(\cdot)$ substituted for $v(\cdot)$ and $v_{\theta}(\cdot)$. In particular, with this change, formula (3.4) describes the finite volume ensembles of $E(\cdot)$.

For the sake of comparison with $v(d E)$, we remark that for $x<y$ :

$$
\int_{\substack{E(x)=u \\ E(y)=v}} v_{J}(d E)=e^{-(y-x) D}(u, v)
$$


with the operator $D$, defined on $L^{2}(\mathbb{R})$ by

$$
(D f)(u)=2 \varrho \frac{d}{d u} f(u)+\frac{\varrho}{e}[f(u-2 e)-f(u)],
$$

replacing the difference operator $\Delta$.

As a consequence of the constraint (4.6), the jump process generated by $e^{-x D}$ is not transitive. However for any fixed $u, v \in 2 e \mathbb{Z}$

$$
e^{-k x_{0} D}(u, v)>0
$$

if

$$
k>(v-u) /(2 e) .
$$

Substituting $v_{J}$ for $v$ in (3.5), with $x=x_{0}$, we obtain a kernel operator $A_{x_{0}}^{(J)}$ on $\ell^{2}(2 e \mathbb{Z})$. Similarly to (3.7), the finite volume expectation value of a function $f(E)$ which depends on $E$ only in the interval $\left[-n x_{0}, m x_{0}\right], n, m \in \mathbb{Z}$ is

$$
\langle f\rangle_{J}^{L}=\frac{\left(\chi_{0},\left(A_{x_{0}}^{(J)}\right)^{L / 2-n} B\left(A_{x_{0}}^{(J)}\right)^{L / 2-n} \chi_{0}\right)}{\left(\chi_{0},\left(A_{x_{0}}^{(J)}\right)^{L} \chi_{0}\right)}
$$

with $B$ given by the kernel:

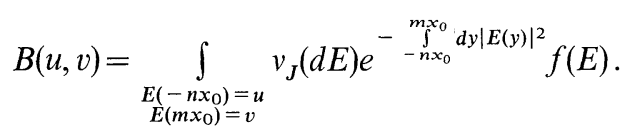

By (4.10) and the estimate (3.10), $A_{x_{0}}^{(J)}$ satisfies the assumptions of the KreinRutman theorem. The claims 1-3 follow now by the application of (3.9) to (4.11).

In order to recover $E(\cdot)$ from $\left\{q_{i}\right\}$ we first note that the finite volume ensembles, and thus also the limiting state, $\langle\ldots\rangle_{J}$, have the symmetry: $E(x) \rightarrow-E(-x)$. Combined with the periodicity, this implies that

$$
\left\langle\int_{0}^{x_{0}} d y E(y)\right\rangle_{J}=0 .
$$

By an application of the ergodic theorem,

$$
\lim _{\substack{|k| \rightarrow \infty \\ k \in \mathbb{Z}}} \frac{1}{|k|} \int_{x}^{x+k x_{0}} d y E(y)=0 \quad \text { for almost every } E(\cdot) .
$$

In fact, by the local integrability of $E$, (4.13) holds also without the restriction $k \in \mathbb{Z}$. The reconstruction formulae (4.7) follow now by the substitution of (4.5) in (4.13).

Proof of Proposition 4. The existence of a limiting state and its periodicity is implied by the stronger statement of Lemma 2.

Equation (4.4) is just the exponentiation of (4.7), which is intended to emphasize the non-invariance of the state, even in its restriction to the particle variables $\left\{q_{i}\right\}$.

Remark. The above proof of translation-symmetry breaking relies on the structural constraint (4.6) which holds irrespectively of the temperature. Thus we regard 
this argument as considerably simpler than those used previously [1,2]. However it leads only to the conclusion that some correlation function is not invariant. We do not offer a simplification of the proof of that statement for the one particle density.

\section{Equilibrium Conditions}

We shall now discuss the structure of the states we have considered from the three points of view which are generally used to locally characterize thermodynamic equilibrium. We shall focus on the two component system. Extension to the jellium requires only the obvious modifications.

\section{a) The Structure of the Conditional Distributions}

In the introduction we have explained the inapplicability of the DLR condition in its usual form. Having gathered more information, we may now complete the analysis of the conditional distribution of the particles in a finite region $\Lambda$, conditioned on the configuration in $\Lambda^{c}$.

For typical configurations, formulae (3.11) and (3.12) permit us to reconstruct the total electric field at the boundary of an interval $\Lambda=[a, b]$ from the location of charges in $\Lambda^{c}$. To be specific, the calculation of $E$ is really carried in three steps:

1) Computing the limit in (3.13) one finds the value of $g(\theta)$ for $\theta=$ external field $\bmod 2 e$.

2) $\langle E\rangle$ is calculated for the state for which the given configuration is "typical", using the inverse function of $g(\theta)$ and an explicit computation of $\langle E\rangle_{\theta}$.

3) Using the above information and the limit in (3.11), (3.12), one finds $E(\cdot)$.

It may be shown that this procedure is equivalent to a more direct, but less explicit, variational method in which one first solves for $E(\cdot)$ up to an addive constant, and then selects the solution which minimizes $\int d x|E(x)|^{2}$.

Thus the local distribution conditioned on the particles in $\Lambda^{c}$ directly corresponds to the local distribution of the electric field $E(\cdot)$, conditioned on $E(\cdot)$ in $\Lambda^{c}$. However, in a sharp contrast with the long range of the Coulomb interaction between particles, $E(\cdot)$ has the Markov property. It implies that the above distribution is given by the corresponding $\left(E_{\ell}, E_{n}\right)$ equilibrium ensemble, introduced in Sect. 3. A striking implication is that the total charge in $[a, b]$ is ${ }^{*}$ restricted to be $Q\left(E_{a}, E_{b}\right)$ [see (3.1)] (i.e. adding a single particle to a "typical" configuration leads to one which is " $a$-typical"). Otherwise the distribution is given by the Gibbs prescription, which is :

$$
\delta\left(\sum_{q_{i} \in \Lambda} \sigma_{i}-\left(E_{\imath}-E_{\ell}\right) / 2\right) \exp \left\{\beta\left[\frac{1}{2} \sum_{q_{i}, q_{j} \in \Lambda} \sigma_{i} \sigma_{j}\left|q_{i}-q_{j}\right|+\sum_{q_{i} \in \Lambda} \sigma_{i} q_{i}\left(E_{\imath}+E_{\ell}\right) / 2\right]\right\} / \text { Norm }
$$

Thus the $\theta$-states satisfy a version of the DLR condition which, with respect to the charge, is canonical rather than grand-canonical (i.e. the $\theta$-states are canonical, in the terminology of [15]). 


\section{b) KMS Condition}

Adding the momentum degrees of freedom, with the independent distribution $\bigotimes_{i}\left(e^{-\beta p_{i} / 2 m} d p_{i} /\right.$ Norm. $)$, we obtain states of an infinite classical system which might be expected to be invariant under the Hamiltonian dynamics. The action of the generator of this time evolution, on local smooth functions $f$, is given by

with

$$
\{H, f\}\left(\left\{q_{i}, p_{i}, \sigma_{i}\right\}\right)=\sum_{k}\left[\frac{p_{k}}{m} \frac{\partial}{\partial p_{k}}+F_{k}\left(\left\{q_{j}, \sigma_{j}\right\}\right) \frac{\partial}{\partial p_{k}}\right] f\left(\left\{q_{i}, p_{i}, \sigma_{i}\right\}\right)
$$

$$
F_{k}\left(\left\{q_{j}, \sigma_{j}\right\}\right)=\lim _{\varepsilon \rightarrow 0} \sigma_{k}\left[E\left(q_{k}+\varepsilon\right)+E\left(q_{k}-\varepsilon\right)\right] / 2,
$$

and $E(\cdot)$ computed by the procedure discussed above. In this setup we may formulate the classical KMS condition [16].

Proposition 5. In each of the $\theta$-states:

$$
\langle\{f, g\}\rangle_{\theta}=\beta\langle f\{H, g\}\rangle_{\theta}
$$

for any pair of local, $C^{2}$, functions $f, g: \Omega \rightarrow \mathbb{R}$. ( $\{$,$\} is the Poisson bracket.)$

Proof. For any finite interval $[a, b]$ let us consider the time evolution for which all the coordinates of the particles outside of $[a, b]$ are kept fixed, and the particles in $[a, b]$ evolve under the Hamiltonian evolution which corresponds to the $\left(E_{\ell}, E_{\hat{\imath}}\right)$ ensemble, with reflecting boundary conditions at $a$ and $b$. Our previous analysis of the conditional distributions implies that the $\theta$-states are invariant under such evolutions and obey the corresponding KMS conditions. These are described by (5.3) restricted to functions which satisfy the reflecting boundary conditions at $a$ and $b$. Choosing $[a, b]$ to strictly contain the domains in which given $f$ and $g$ are measurable we have a proof of the claim.

\section{c) $B B G K Y$ Hierarchy}

Let $\left\{\varrho_{\theta}^{(n)}\right\}$ be the correlation functions of a $\theta$-state. We shall now see that they obey the following form of the $B B G K Y$ hierarchy of equations:

$$
\begin{aligned}
& \frac{\partial}{\partial q_{i}} \varrho_{\theta}^{(n)}\left(\left\{q_{k}, \sigma_{k}\right\}_{k=1, \ldots, n}\right) \\
& =\beta\left[\langle E\rangle_{\theta} \sigma_{i}+\sum_{j(\neq i)} F_{i, j}\right] \varrho_{\theta}^{(n)}\left(\left\{q_{k}, \sigma_{k}\right\}_{k=1, \ldots, n}\right) \\
& \quad+\beta \lim _{y \rightarrow \infty} \sum_{\sigma_{n+1}= \pm e} \int_{-y}^{y} d q_{n+1} F_{i, n+1} \varrho_{\theta}^{(n+1)}\left(\left\{\left(q_{k}, \sigma_{k}\right)\right\}_{k=1, \ldots, n+1}\right)
\end{aligned}
$$

with

$$
F_{i j}=\sigma_{i} \sigma_{j} \operatorname{sgn}\left(q_{i}-q_{j}\right) .
$$

The special features of (5.4), in comparison with the $B B G K Y$ hierarchy for short range systems, are the use of the limit $y \rightarrow \infty$ and the term with $\langle E\rangle_{\theta}$. The significance of this term was discussed in [17]. 
To prove (5.4), we observe that for any $a, b \in(-\infty, \infty)$ the invariance of $\langle\ldots\rangle_{\theta}$, (with the added Boltzmann distribution of momenta) under the modified dynamics discussed in the proof of Proposition 5, implies the following set of equations:

$$
\begin{aligned}
& \frac{\partial}{\partial q_{i}} \varrho_{\theta}^{(n)}\left(\left\{q_{k}, \sigma_{k}\right\}_{k=1, \ldots, n}\right) \\
& =\beta\left[\left\langle[E(a)+E(b)] / 2 \mid\left\{q_{k}, \sigma_{k}\right\}_{k=1, \ldots, n}\right\rangle_{\theta} \sigma_{i}+\sum_{j(\neq i)} F_{i, j}\right] \varrho_{\theta}^{n}\left(\left\{q_{k}, \sigma_{k}\right\}_{k=1, \ldots, n}\right) \\
& \quad+\beta \sum_{\sigma_{n+1}= \pm e} \int_{a}^{b} d q_{n+1} F_{i, n+1} \varrho_{\theta}^{n+1}\left(\left\{q_{k}, \sigma_{k}\right\}_{k=1, \ldots, n+1}\right)
\end{aligned}
$$

for $\left\{q_{k}\right\}_{k=1, \ldots, n} \in(a, b)$. Here $\left\langle\cdot \mid\left\{\left(q_{k}, \sigma_{k}\right)\right\}\right\rangle_{\theta}$ is the state conditioned on the existence of the charges $\left\{\sigma_{k}\right\}$ at $\left\{q_{k}\right\}_{k=1, \ldots, n}$. Alternatively, one may derive (5.5) using the explicit formula (5.1).

The mixing property of $\langle\ldots\rangle_{\theta}$ implies that for any given $\left\{\left(q_{k}, \sigma_{k}\right)\right\}$

$$
\lim _{|a|,|b| \rightarrow+\infty}\left\langle[E(a)+E(b)] / 2 \mid\left\{\left(q_{k}, \sigma_{k}\right)\right\}\right\rangle=\langle E\rangle_{\theta} .
$$

Substituting (5.6) in (5.5) we obtain (5.4), in the limit $a \rightarrow-\infty, b \rightarrow \infty$.

\section{Remarks on the Phenomenology}

The $\theta$-states can be viewed as limiting states of finite, neutral systems placed in a condenser. In the corresponding electrostatic terminology the applied field is the electric displacement $D$. At any temperature and density, the average field, $\langle E\rangle(D)$, is a non-trivially periodic function of $D$ depending only on $\theta=D \bmod 2 e$, with $\langle E\rangle(-D)=-\langle E\rangle(D)$ and $\langle E\rangle(D=e)=0$.

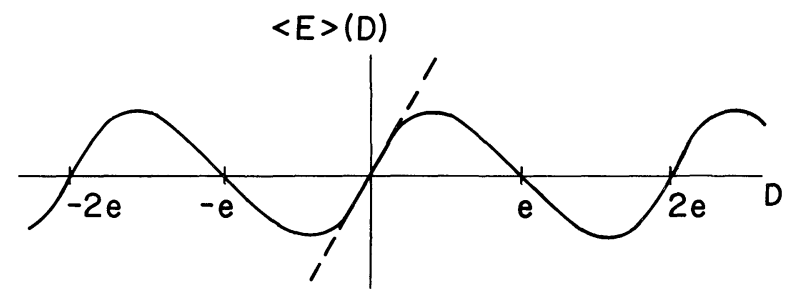

Fig. 3. The effective field as function of the applied field (schematically)

The dielectric constant $\varepsilon=\left(\left.\frac{d\langle E\rangle(D)}{d D}\right|_{D=0}\right)^{-1}$ is finite at all temperatures and densities (the one-dimensional Coulomb system is an insulator, see [18] for a discussion of this point).

If $Q(x)$ and $P(x)=x Q(x)$ denote the charge and polarization density in a finite neutral $\left(E_{\imath}=E_{\ell}=D\right)$ equilibrium ensemble in $[-L, L]$, the total polarization $P_{L}$ is given by

$$
P_{L}=\frac{1}{2 L} \int_{-L}^{L} P(x) d x=\frac{1}{4 L} \int_{-L}^{L} x \frac{d}{d x} E(x) d x=1 / 2\left(D-\frac{1}{2 L} \int_{-L}^{L} E(x) d x\right) .
$$


Therefore we get in the thermodynamic limit the usual phenomenological relation

$$
\langle P\rangle(D)=\lim _{L \rightarrow \infty}\left\langle P_{L}\right\rangle^{L}(D)=1 / 2(D-\langle E\rangle(D)) .
$$

For small values of the plasma parameter $\frac{\varrho}{\beta e^{2}}$, and $0 \leqq D<e$, the system can be viewed as a collection of dipoles which interact only when overlapping. The binding potential of a $(+-)$ pair in the $D$ field is $V_{D}(x)=e|x|+D x, 0 \leqq D<e$. For $D=e, V_{D}(x)$ is no more confining and the dipoles disassociate. It is easily checked that for $0 \leqq D \leqq e$ the equation of state at low density is that of a free gas of binary molecules (i.e. $\beta p=1 / 2 \varrho, \varrho \rightarrow 0$ ) whereas for $D=e$ we have a free gas of particles (i.e. $\beta p=\varrho, \varrho \rightarrow 0)$. When $e<D<2 e$, one $(+-)$ pair is broken; the corresponding charges go to boundary and over screen the applied field, hence the change of sign of $\langle E\rangle(D)$ at $D=e$. For $D=2 e$, this surface charge shields the applied field exactly, and therefore the $D=2 e$ state is identical to the $D=0$ state. As $D$ increases the same mechanism repeats: Whenever the value of $D$ passes an odd multiple of $e$, another dipole disassociates, and the surface charge increase by one unit. However just at these odd values of $D / e$ each of the dipoles is unstable.

The above discussion in terms of dipoles is meaningful only when the length of a dipole is smaller than the dipole separation. Although there is no sharp phase transition, in the plasma limit, $\frac{\varrho}{\beta e^{2}} \gg 1$, dipoles overlap and typical configurations look qualitatively completely different.

Acknowledgements. We wish to thank Professor Ph. Choquard and Professor E. H. Lieb for kind hospitality extended to M. A. at Ecole Polytechnique Fédérale de Lausanne and, respectively, to Ph. M. at Princeton University.

\section{References}

1. Kunz, H.: Ann. Phys. 85, 303 (1974)

2. Brascamp, H.J., Lieb, E.H.: In: Functional integration and its applications. A.M. Arthurs (ed.). Clarendon Press 1975

3. Edwards, S.F., Lenard, A.: J. Math. Phys. 3, 778 (1962)

4. Lenard, A.: J. Math. Phys. 4, 533 (1963)

5. Fröhlich, J., Park, Y.M.: Commun. Math. Phys. 57, 235 (1978)

6. Lanford, O.E.: In: Dynamical systems, theory, and applications. Lecture Notes in Physics, Vol. 38. Berlin, Heidelberg, New York: Springer 1975

7. Dobrushin, R.L.: Theory Probab. Its Appl. 13, 197 (1968)

8. Lanford, O.E., Ruelle, D. : Commun. Math. Phys. 13, 194 (1969)

9. Martin, Ph., Yalcin, T.: J. Stat. Phys. 22, 435 (1980)

10. Metha, M.L., Dyson, F.J.: J. Math. Phys. 4, 713 (1963)

11. Simon, B.: Functional integration and quantum mechanics. New York: Academic Press 1979

12. Reed, M., Simon, B.: Methods of modern mathematical physics, Vol. IV, Sect. XIII.16. New York: Academic Press 1978

13. Krein, M.G., Rutman, M.A.: Trans-Amer. Math. Soc. 26, 199 (1950) (Th $\beta^{\prime \prime}$, p. 279)

14. Aizenman, M., Fröhlich, J. : In preparation

15. Aizenman, M., Goldstein, S., Lebowitz, J.: Commun. Math. Phys. 62, 279 (1978)

16. Gallavotti, G., Verboven, E.: Nuovo Cimento 28, 274 (1975), and Aizenman, M., Goldstein, S., Gruber, Ch., Lebowitz, J., Martin, Ph. : Commun. Math. Phys. 53, 209 (1977) 
17. Gruber, Ch., Lugrin, Ch., Martin, Ph.: Helv. Phys. Acta 51, 829 (1978) and J. Stat. Phys. 22, 193 (1980)

18. Zittartz, J.: Z. Physik B31, 63 and 79 (1978)

Communicated by A. Jaffe

Received May 22, 1980 